\title{
Kernos
}

Revue internationale et pluridisciplinaire de religion grecque antique

$20 \mid 2007$

Varia

\section{Thesaurus cultus et rituum antiquorum (ThesCRA)}

\section{Corinne Bonnet}

\section{(apenEdition \\ Journals}

\section{Édition électronique}

URL : https://journals.openedition.org/kernos/409

DOI : $10.4000 /$ kernos.409

ISSN : 2034-7871

\section{Éditeur}

Centre international d'étude de la religion grecque antique

\section{Édition imprimée}

Date de publication : 1 janvier 2007

Pagination : 458-462

ISSN : 0776-3824

Référence électronique

Corinne Bonnet, "Thesaurus cultus et rituum antiquorum (ThesCRA) », Kernos [En ligne], 20 | 2007, mis en ligne le 17 mars 2011, consulté le 08 septembre 2022. URL : http://journals.openedition.org/ kernos/409; DOI : https://doi.org/10.4000/kernos.409 
Thesaurus cultus et rituum antiquorum (ThesCRA), Los Angeles, The J. Paul Getty Museum. 5 vols in- $4^{\circ}$. I (2004) XIX +672 p., 139 p. de planches; II (2004) XXI+646 p., 117 p. de planches; III (2005) XVIII+434 p., 72 p. de planches; IV (2005) XIV+485 p., 60 p. de planches; V (2005) XIX+502 p., 67 p. de planches. ISBN : 10 0-89236-787-3.

Une Préface signée par V. Lambrinoudakis et une Introduction due à la plume de J. Boardman éclairent les objectifs et les modalités de mise en œuvre des cinq volumes qui composent le ThesCRA. Mis en chantier cinq ans après la publication du dernier volume du Lexicon Iconographicm Mythologiae Classicae (LIMC), le ThesCRA participe d'une même philosophie, s'appuie sur les ressources archivistiques ainsi constituées et s'enracine dans les mêmes réseaux de collaboration, au niveau des comités et des équipes. Voici comment J. Boardman définit l'objet de cet instrument de travail : « It sets out to present scholars with a comprehensive account of all susbtantial aspects of Greek, Roman and Etruscan religion, apart from any assessment of the purely spiritual or philosophical, and only incidentally with the historical. » Le ThesCRA ambitionne donc, dans le projet de ses concepteurs, de proposer une série de mises au point prenant en compte l'ensemble des sources, archéologiques, iconographiques, épigraphiques et littéraires.

Deux niveaux sont à distinguer, apprend-on encore dans l'Introduction, dans le traitement du sujet : un niveau dynamique, qu'explorent les trois premiers volumes, et un niveau statique, couvert par les deux derniers volumes. La pratique cultuelle relève de la première section; les lieux, le personnel et les instruments de culte sont inclus dans la seconde. On nous annonce, par ailleurs, un troisième niveau à venir, qui concernera le comportement religieux.

Comme pour le LIMC, mais dans une moindre mesure, les divers blocs thématiques, que nous allons détailler ci-dessous, placés sous la responsabilité d'un ou de plusieurs spécialistes, ont fait l'objet d'une certaine modélisation dans le traitement, nous y reviendrons. Disons déjà, de manière quelque peu schématique, que chaque notice comprend à la fois une introduction (historiographie, définition, vocabulaire, sources, etc.), un corps présentant la documentation, sous forme d'une liste de testimonia numérotés selon un classement thématique, enfin une éventuelle conclusion. Le catalogue des testimonia a pour finalité d'illustrer le propos par des renvois aux principales traces, de toute nature, des phénomènes décrits. Ce recueil de sources est, cependant, selon les cas et sans que l'on comprenne nécessairement pourquoi, tantôt fortement sélectif, tantôt à vocation d'exhaustivité. Pour les sources iconographiques, en particulier, on a favorisé les renvois au matériel figurant dans les notices du LIMC et un riche dossier de plus de cent planches en noir et blanc termine chaque volume. On précisera, enfin, que la plupart des thèmes sont successivement traités dans le monde grec, étrusque et romain. Occasionnellement, la documentation chypriote ou procheorientale est mise en évidence de manière autonome, d'une façon qui semble bien arbitraire : pourquoi consacrer une section au banquet proche-oriental (II, p. 251-254) qui ne me semble pas plus déterminant pour le monde gréco-romain que le sacrifice humain, la divination ou l'apothéose?

$\mathrm{Au}$ lecteur qui pourrait légitimement s'interroger sur le découpage spatio-temporel, J. Boardman répond, en précisant que les documents remontant à l'âge du Bronze ne sont admis que comme prémisses de la religion de l'âge du Fer, tandis que, en aval, les pratiques chrétiennes ne sont pas prises en compte. Par ailleurs, la focale est placée sur le monde classique, dans une moindre mesure sur la civilisation étrusque et accessoirement sur les cultures périphériques. 
On évitera de se prononcer d'emblée sur la validité de ces choix, que l'on mettra plutôt à l'épreuve des faits, mais en revanche, on exprimera quelque perplexité quant à l'articulation, bien théorique et quelque peu superficielle ou conventionnelle, entre un niveau dynamique et un niveau statique de la religion. En effet, peut-on considérer le sacrifice comme dynamique et les prêtres comme statiques, alors que l'un se conçoit difficilement sans les autres? Sans doute ne faut-il pas attribuer trop d'importance à ce cadre un peu bancal et convient-il avant tout de s'attacher à la substance des notices.

Précisons, tout d'abord, la liste des notices, sans mentionner les noms des collaborateurs, vraiment trop nombreux pour figurer tous ici. Il suffira de dire que les meilleurs spécialistes du domaine ont pris part à la monumentale entreprise qui, comme le LIMC, recourt à quatre langues, anglais, français, allemand et italien.

\section{Volume I}

1. Processions

2.a. Sacrifices

2.b. Libations

2.c. Fumigations

2.d. Offrandes votives

\section{Volume III}

6.a. Divination

6.b. Prière, gestes et actes de prière

6.c. Gestes et actes de vénération

6.d. Hikésie

6.e. Asylie

6.f. Serment

6.g. Malédiction

6.h. Profanation

6.i. Magie

Addendum au vol. II

3.b. Consécration, rites de fondation

\section{Volume II}

3.a. Purification

3.b. Consécration, rites de fondation

3.c. Initiation

3.d. Héroïsation et apothéose

4.a. Banquet

4.b. Danses

5. Rites et activités relatifs aux images de culte

\section{Volume IV}

1.a. Lieux de culte

1.b. Représentations de lieux de culte

Volume V

2.a. Personnel de culte

Il est naturellement impossible de rendre compte ici de l'ensemble de l'immense matière traitée dans cette ambitieuse entreprise collective, dont on saluera sans plus tarder l'extrême utilité. On proposera dès lors quelques réflexions d'ensemble, d'une part, et une série d'observations ponctuelles, d'autre part, afin d'éclairer les lecteurs sur l'usage, ou mieux les usages possibles de ce qui s'est déjà imposé, dans la lignée du LIMC, comme un instrument de travail fondamental sur les religions, et au-delà sur les sociétés, de l'Antiquité. Sur le plan de la méthode, il soulève une question de fond, à savoir est-il légitime d'appliquer l'approche « sérielle » déployée dans le LIMC aux pratiques religieuses ? Celles-ci se laissent-elles sérier et étiqueter aussi aisément que les représentations iconographiques? La démarche mérite réflexion. Une réponse univoque ne peut être apportée et, au final, l'essai s'avère fructueux, quoique, à divers égards, problématique.

Comme toute entreprise collective, le ThesCRA est tributaire de la qualité du travail effectué par ses divers collaborateurs. Il s'agit de très bons spécialistes, mais il semble assez évident que tous n'ont pas atteint, sans doute aussi en raison des délais qui leur ont été impartis, le même degré d'approfondissement dans le repérage et dans l'exploitation des sources. La valeur des notices est donc inégale, mais dans l'ensemble excellente, et l'amal- 
game entre les diverses portions de notices est plutôt réussi, en dépit de certaines légères disharmonies. Il faut, me semble-t-il, au-delà des limites et des imperfections, saluer l'extraordinaire utilité d'un tel instrument de travail que bien d'autres domaines de la recherche (je pense ainsi aux études orientales) pourraient envier.

Une difficulté générale mérite qu'on s'y arrête, à savoir celle d'isoler - même si l'on fait l'impasse sur la curieuse dichotomie religion dynamique / religion statique évoquée cidessus - ce qui est spécifiquement religieux et, au sein de cette catégorie, de dégager des «lemmes » autonomes. Il y a longtemps, en effet, que l'on a souligné la profonde imbrication des niveaux civiques et religieux dans les civilisations anciennes. C'est pourquoi, sans doute, dans la notice "processions» qui inaugure le volume I, au sein de la section étrusque, S. Bruni prend en compte les défilés de magistrats à pied, sur bige ou quadrige, en dépit du fait que le lien direct avec la vie religieuse n'est guère explicite. Une telle vision englobante pourrait amener, si on voulait pousser la logique dans ses derniers retranchements, à rassembler une documentation gigantesque, pratiquement toute la documentation antique, dans la mesure où le religieux est constamment sous-jacent. Les frontières assignées au thème étudié (serment, offrande, etc.) et à la documentation pertinente relèvent donc d'un choix conceptuel et pratique que les Auteurs n'explicitent pas toujours. Ainsi, la notice «Gesti e atti di venerazione » ne fait l'objet d'aucune définition préliminaire, de sorte que la catégorie se détache, comme en creux, par rapport aux autres notices apparentées (prière, offrande, sacrifice, supplication, etc.).

Bref, on perçoit, comme c'est bien naturel dans une publication d'une telle ampleur, des oscillations dans la manière d'appréhender et de circonscrire le sujet, ce qui est, en ultime instance, révélateur du fonctionnement des sociétés anciennes encore "enchantées». Personne, je crois, ne lira d'un bout à l'autre le Thes $C R A$, mais une vision d'ensemble suffit à dessiner les contours fluides et complexes de pratiques et de croyances à géométrie variable. Sur ce point, l'intention affichée dans l'Introduction de traiter la religion en dehors de toute considération spirituelle, philosophique, voire même historique tient d'un parti pris à la fois naïf et infondé. On comprend bien le souci de se libérer de toute approche confessionnelle, mais la religion n'est pas un conglomérat de phénomènes, de manifestations ou de pratiques; elle s'enracine dans des croyances et dans des contextes. Pour le dire avec F. Cumont, écrivant à S. Reinach en 1908, il faut veiller à ne pas traiter « la religion comme un squelette»; or, "c'est, non pas une pièce d'anatomie, mais une force vive qui agit dans l'histoire de l'humanité ». La nécessité de répartir la documentation au sein d'une sorte de «typologie» (comme dans le LIMC) des manifestations religieuses donne aux notices une allure quelque peu " phénoménologique » qui brouille les logiques chronologiques et géographiques et qui rend peu perceptibles les variations spatio-temporelles.

Prenons comme exemple la notice «sacrifices». Elle s'ouvre sur le monde grec, avec une bonne introduction historiographique et lexicographique, catégories de sacrifices et choix opérés dans la notice, à savoir de privilégier les sacrifices sanglants. Choix qui peut se justifier, mais qui semble à contre-courant par rapport aux tendances récentes de la recherche ( $c$. S. Georgoudi - R. Koch Piettre - F. Schmidt [éds], La cuisine et l'autel: les sacrifices en questions dans les sociétés de la Méditerranée ancienne, Turnhout, 2005) où est soulignée la solidarité profonde entre offrandes animées et inanimées. Les entrées «libation », "fumigations » et «offrandes votives » ne complètent du reste qu'imparfaitement le tableau sur ce point. Le sacrifice sanglant est ensuite analysé quant à son origine, son déroulement, ses traces archéologiques; puis vient un catalogue de sacrifices par divinité et héros, bien intéressant (p. 68-95), suivi d'une étude sur la typologie des victimes et d'une analyse par circonstance et lieu de sacrifice (guerre, purification, maison, etc.). Enfin, on décompose les diverses phases du sacrifice et de sa cuisine. Le sacrifice humain, en bout de notice, reçoit un 
traitement plus rapide (129-134), voire trop rapide. Les deux sections consacrées au sacrifice en milieu étrusque et en milieu romain suivent une logique analytique similaire.

Au final, on apprend beaucoup de choses, on a accès à une documentation considérable, soigneusement ordonnée, mais il faut savoir que certaines questions restent sans réponse ou de réponse difficile. Par exemple, impossible, ou presque, de savoir ce qui distingue la pratique sacrificielle archaïque de la pratique classique ou hellénistique. Pas plus simple de mesurer les écarts entre les façons de faire en métropole ou en domaine colonial, à Rome ou en provinces. L'approche typologico-descriptive qui est privilégiée (avec les éléments constitutifs, les divinités concernées, les modes de représentation, les moments et les lieux, les acteurs, etc.) tend à aplatir sur un unique horizon (anihistorique ? transhistorique ? métahistorique ?) ce qui, dans les faits, correspond à une infinité de variantes chevillées dans des contextes spatio-temporels déterminés. L'historien des religions restera donc parfois sur sa faim, dans la mesure où la casuistique n'aide pas nécessairement à comprendre comment et c'est un principe méthodologique essentiel - les pratiques religieuses se forment dans un rapport dialectique avec des contextes historiques, culturels, sociaux, économiques toujours singuliers. Ainsi, que l'on ait recourt, pour les rites de purifications (II, p. 19 sq.), à une série de médiateurs aussi variés que l'eau, le sang, le sel, l'or, le feu ou d'autres agents encore, au demeurant souvent combinés, comme on nous le précise d'emblée, montre que la qualité cathartique n'est pas inhérente à tel ou tel élément, mais qu'elle est culturellement construite pas les sociétés en fonction de codes symboliques spécifiques et variables selon les lieux et les époques, selon l'architecture symbolique des groupes concernés.

À cet égard, le ThesCRA pourra bien entendu susciter une série d'enquêtes historiques de ce type, dans la mesure où il facilitera l'accès aux sources et favorisera une démarche comparative. Celle-ci est, en effet, implicitement présente dans les cinq volumes : comparaison entre aires culturelles, comparaison au sein de chaque aire; elle n'est guère déployée, ni soulignée, mais elle n'attend, en quelque sorte, que le regard fureteur du comparatiste pour émerger de ce recueil de données.

Paradoxalement peut-être, les tomes IV et V, consacrés aux prétendus aspects statiques de la religion (lieux et personnel de culte), constituent des répertoires particulièrement réussis et précieux. L'approche archéologique se déploie avec beaucoup de finesse et d'efficacité dans la typologie des lieux de culte, de leur usage et de leur représentation. Cette partie constitue, à mon sens, un modèle du genre et pourrait vraiment inspirer les spécialistes des autres aires culturelles. Ici, l'effort typologique est particulièrement éclairant et fructueux. Pour le personnel de culte, c'est davantage la documentation épigraphique et iconographique qui est sollicitée, les unes et les autres étant mises en œuvre pour la dernière section, fort intéressante, mais aussi très foisonnante, consacrée aux "instruments du culte ", avec une vaste typologie d'objets, dont l'utilisation tout court et l'utilisation cultuelle en particulier ne sont pas toujours assurées, mais qui offrent un panorama intéressant de certains aspects de la religion au quotidien, dans son fonctionnement "mécanique (vaisselle, mobilier, éclairage, attributs, etc.), bref tout ce qui « habillait» et « animait» les sanctuaires, peuplés de dieux et de prêtres, de fidèles et d'objets.

On attend avec impatience le volume d'index qui s'impose absolument pour permettre aux lecteurs, quel que soit leur niveau d'utilisation du Thes $C R A$, de s'orienter dans le foisonnement étourdissant de données et de références. On espère, de même, comme l'annonce J. Boardman dans l'Introduction, qu'une partie substantielle des archives photographiques sera bientôt disponible sur la toile. Le Thes $C R A$, comme son grand frère le $L I M C$, est appelé à devenir, ou plutôt est déjà, un instrument de travail irremplaçable et incontournable. On saura donc vivement gré au comité qui a pris en charge cette lourde entreprise collective et aux auteurs qui ont rassemblé et valorisé une documentation si considérable. La structure du 
Thes $C R A$ nous est apparue comme un peu rigide, non sans quelques éléments d'incohérence, et surtout insuffisamment attentive aux ancrages historiques des religions anciennes, mais elle invite à une multiplicité d'usages et de questionnements, ciblés, croisés, comparés, qui contribueront assurément à revivifier les études dans ce domaine.

Corinne Bonnet

(Université de Toulouse II - Le Mirail)

DETIENNE Marcel, Les jardins d'Adonis. La mythologie des parfums et des aromates en Grèce. Suivi d'une interprétation de Jean-Pierre Vernant et d'une lecture de Claude Lévi-Strauss. Nouvelle édition, augmentée d'un après-propos de l'auteur, Paris, Gallimard, 2007. 1 vol. $10,5 \times 18 \mathrm{~cm}, 350$ p. (folio histoire). ISBN : 978-2-07-034183-2.

IDEM, Les dieux d'Orphée, Paris, Gallimard, 2007. 1 vol. 10,5 × 18 cm, 238 p. (folio histoire). ISBN : 978-2-07-034182-5.

On ne présente plus ces deux ouvrages, et en particulier le premier qui passe à juste titre pour être une des applications les plus intelligentes et les plus fécondes d'une méthode structurale. Chacun se réjouira qu'une nouvelle édition les rende plus aisément accessibles.

La première édition des Jardins d'Adonis date de 1972; la réédition de 1989 comprenait déjà, outre les deux textes de Vernant et de Lévi-Strauss rappelés ci-dessus, une postface de l'auteur. La présente édition reproduit le tout et y ajoute donc un intéressant «aprèspropos» (p. 261-269) intitulé «Quel est donc le sexe de la mythologie ?», qui invite à tempérer certaines ardeurs interprétatives. Avec un index rerum très copieux.

Les dieux d'Orphée est un nouveau titre donné à l'ouvrage L'écriture d'Orphée, paru en 1989, mais qui se voit amputé ici de six brèves études, pour lesquelles l'A. ne sollicite, dit-il avec humour, «ni fleurs, ni couronnes ». À noter, pour mieux comprendre l'« après-propos » du livre précédent, que le dernier et copieux chapitre s'intitule «Du sexe de la mythologie». Avec un index des noms d'auteurs anciens et modernes.

André Motte

(Université de Liège)

\section{Actes de colloques et ouvrages collectifs}

Anello Pietrina, MARTorana Giuseppe, SAmmarTano Roberto (éds), Ethne e religioni nella Sicilia antica. Atti del convegno (Palermo, 6-7 dicembre 2000), Roma, G. Bretschneider Editore, 2006 (Kokalos, suppl. 18)

M. MAZZA, Identità e religioni: considerazioni introduttive, p. 1-22; S. TUSA, Considerazioni sulla religiosità delle popolazioni pre-elleniche siciliane tra il Paleolitico Superiore et l'Età del Bron₹o, p. 23-41; R.M. ALBANESE PROCELli, Pratiche religiose in Sicilia tra protostoria e arcaismo, p. 43-70; P. ANELLO, Ciclopi e Lestrigoni, p. 7185; G. TERrANOva, Apprestamento cultuale in una tomba castellucciana a prospetto pilastrato dell'area iblea, p. 87-106; D. Pancucci, I Sicani, p. 107-119; N. Cusumano, Siculi, p. 121-145; St. De Vido, Gli Elimi, p. 147-179; C. JOURDAIN-ANNEQuIN, I Greci - Les Grecs, p. 181-203; C. BONNET, La religione fenicia e punica in Sicilia, p. 205-216; I. CHIRASSI COLOMBO, La Sicilia e l'immaginario romano, p. 217-249; G. SFAMENi GASPARRO, I culti orientali nella Sicilia ellenistico-romana, p. 251-328; S.N. CONSOLO LANGHER, Religione e regalità. Tra Grecia, Oriente e Sicilia: fondamenti ideologici e politici nel culto del sovrano ellenistico, p. 329-342; V. LOMBINO, Identità del primo cristianesimo siciliano (Secoli III-VI), p. 343-393; 